QUARTERLY OF APPLIED MATHEMATICS

VOLUME LXV, NUMBER 1

MARCH 2007, PAGES 155-162

S 0033-569X(07)01029-0

Article electronically published on February 12, 2007

\title{
THE STUDY OF DYNAMIC BEHAVIOR OF FUNCTIONALLY GRADED PIEZOELECTRIC MATERIALS AND AN APPLICATION TO A CONTACT PROBLEM
}

\author{
BY \\ B. M. SINGH (Department of Computer Science, The University of Calgary, Calgary, Alberta, \\ Canada T2N-1N4), \\ J. ROKNE (Department of Computer Science, The University of Calgary, Calgary, Alberta, Canada \\ T2N-1N4), \\ AND
}

R. S. DHALIWAL (Department of Mathematics and Statistics, The University of Calgary, Calgary, Alberta, Canada T2N-1N4)

\begin{abstract}
In the present paper, the dynamic behavior of functionally graded piezoelectric materials is investigated when it is under anti-plane mechanical loading and in-plane electrical loading. It is assumed that the shear modulus, the piezoelectric modulus, the dielectric modulus and mass density of FGPM vary continuously as functions of $X$ and $Y$. By using Fourier transforms the solution of equilibrium equations is obtained in closed form. The expressions for displacement and electrical potential are obtained in terms of one unknown function. Finally the results are applied to obtain a solution of the moving contact problem on the surface of the functionally graded piezoelectric material (FGPM).
\end{abstract}

1. Introduction. It is well known that piezoelectric materials produce an electric field when deformed and undergo deformation when subjected to an electric field. Due to this intrinsic coupling effect between the mechanical and electrical fields, piezoelectric materials have been extensively used in electromechanical devices such as sensors, filters, ultrasonic generators and actuators. The demand for piezoelectric materials with high strength, high toughness, low thermal expansion coefficient and low dielectric constant encourages the study of functionally graded piezoelectric materials [1, 2]. To meet the demand of advanced piezoelectric materials and with the help of modern material processing technology, the concept of functionally graded materials has recently been extended in piezoelectric materials [3-[10]. In recent years some studies of propagating

Received April 14, 2006.

2000 Mathematics Subject Classification. Primary 74A30.

E-mail address: rokne@cpsc.ucalgary.ca

E-mail address: dhali.r@shaw.ca

(C)2007 Brown University 
crack problems in homogeneous piezoelectric materials have been conducted by various authors [1]-17.

There have been a number of studies devoted to the theoretical analysis and engineering applications of piezoelectric materials in references [18-20].

Most of the work has been done on crack problems, and it is worth mentioning that some work on inclusion problems in piezoelectric medium may be found in references 21]-24. In most of the above references, the authors considered the crack problems in functionally graded materials in which non-homogeneity vary perpendicular to the crack and that the non-homogeneity vary in only one direction.

The main object of this problem is to explore the FGPM, when the material properties change in both $X$ and $Y$ directions. In this case the solution of equilibrium equations is obtained analytically in closed form. As an application the closed form solution is used to obtain a solution to the problem of a moving load on the surface of a piezoelectric material.

2. Governing equations and solution of equilibrium equations. Let $X, Y$ and $Z$ denote regular Cartesian coordinates, where the $Z$-axis orients in the poling direction of a poled piezoelectric composite. In the case of the out-of-plane displacement component and the in-plane-electric components, the governing equations simplify to:

$$
\begin{aligned}
& u_{X}=u_{Y}=0, u_{Z}=U_{Z}(X, Y, t), \\
& E_{X}=E_{X}(X, Y, t), \quad E_{Y}=E_{Y}(X, Y, t) \quad E_{Z}=0,
\end{aligned}
$$

where the electric field intensities $E_{X}$ and $E_{Y}$ are related to the electric potential $\Phi$ by the following equations:

$$
E_{X}=-\frac{\partial \Phi}{\partial X}, \quad E_{Y}=-\frac{\partial \Phi}{\partial Y} .
$$

Assuming that the shear modulus, the piezoelectric modulus and dielectric modulus are functions of $X$ and $Y$, the constitutive equations for FGPM are

$$
\begin{aligned}
& \sigma_{X Z}(X, Y, t)=c_{44}(X, Y) \frac{\partial u_{Z}}{\partial X}+e_{15}(X, Y) \frac{\partial \Phi}{\partial X}, \\
& \sigma_{Y Z}(X, Y, t)=c_{44}(X, Y) \frac{\partial u_{Z}}{\partial Y}+e_{15}(X, Y) \frac{\partial \Phi}{\partial Y}, \\
& D_{X}=e_{15}(X, Y) \frac{\partial u_{Z}}{\partial X}-\lambda_{11}(X, Y) \frac{\partial \Phi}{\partial X} \\
& D_{Y}=e_{15}(X, Y) \frac{\partial u_{Z}}{\partial Y}-\lambda_{11}(X, Y) \frac{\partial \Phi}{\partial Y}
\end{aligned}
$$

where $\sigma_{X Z}$ and $\sigma_{Y Z}$ are the shear stresses while $D_{X}$ and $D_{Y}$ are electric displacements and $c_{44}(X, Y), e_{15}(X, Y)$ and $\lambda_{11}(X, Y)$ are shear modulus, piezoelectric modulus and dielectric modulus respectively. In the following we use the notation

$$
c_{44}=c_{44}(X, Y), \quad e_{15}=e_{15}(X, Y), \quad \lambda_{11}=\lambda_{11}(X, Y) .
$$

The equilibrium equations are:

$$
\frac{\partial}{\partial X} \sigma_{X Z}+\frac{\partial}{\partial Y} \sigma_{Y Z}=\rho \frac{\partial^{2} u}{\partial t^{2}}, \frac{\partial}{\partial X} D_{X}+\frac{\partial}{\partial Y} D_{Y}=0 .
$$


Making use of equations (2.4)-(2.7) we can write equations (2.9) in the form:

$$
\begin{aligned}
& c_{44} \nabla^{2} u_{Z}+\frac{\partial c_{44}}{\partial X} \frac{\partial u_{Z}}{\partial X}+\frac{\partial c_{44}}{\partial Y} \frac{\partial u_{Z}}{\partial Y}+e_{15} \nabla^{2} \Phi+\frac{\partial \Phi}{\partial Y} \frac{\partial e_{15}}{\partial Y}+\frac{\partial e_{15}}{\partial X} \frac{\partial \Phi}{\partial X} \\
& =\rho \frac{\partial^{2} u}{\partial t^{2}}, \\
& e_{15} \nabla^{2} u_{Z}+\frac{\partial e_{15}}{\partial X} \frac{\partial u_{Z}}{\partial X}+\frac{\partial e_{15}}{\partial Y} \frac{\partial u_{Z}}{\partial Y}-\lambda_{11} \nabla^{2} \Phi-\frac{\partial \lambda_{11}}{\partial X} \frac{\partial \Phi}{\partial X}-\frac{\partial \lambda_{11}}{\partial Y} \frac{\partial \Phi}{\partial Y} \\
& =0,
\end{aligned}
$$

where $\rho$ denotes the density of the material.

We assume that the shear modulus, piezoelectric modulus and dielectric modulus are in the following form:

$$
\left(c_{44}, e_{15}, \lambda_{11}, \rho\right)=\left(c_{44}^{0}, e_{15}^{0}, \lambda_{11}^{0}, \rho_{0}\right) p(X) q(Y),
$$

where $c_{44}^{0}, e_{15}^{0}, \lambda_{11}^{0}$ and $\rho_{0}$ are material constants. Substituting from equations (2.12) into equations (2.10) and (2.11) we find that

$$
\begin{aligned}
& {\left[\nabla^{2} u_{Z}+\frac{1}{p(X)} \frac{d p}{d X} \frac{\partial u_{Z}}{\partial X}+\frac{1}{q} \frac{d q}{d Y} \frac{\partial u_{Z}}{\partial Y}\right]} \\
& +\left(\frac{e_{15}^{0}}{c_{44}^{0}}\right)\left[\nabla^{2} \Phi+\frac{1}{p(X)} \frac{d p}{d X} \frac{\partial \Phi}{\partial X}+\frac{1}{q} \frac{d q}{d Y} \frac{\partial \Phi}{\partial X}\right]=\left(\frac{\rho_{0}}{c_{44}^{0}}\right) \frac{\partial^{2} u}{\partial t^{2}}, \\
& {\left[\nabla^{2} u_{Z}+\frac{1}{p(X)} \frac{d p}{d X} \frac{\partial u_{Z}}{\partial X}+\frac{1}{q} \frac{d q}{d Y} \frac{\partial u_{Z}}{\partial Y}\right]} \\
& -\left(\frac{\lambda_{11}^{0}}{e_{15}^{0}}\right)\left[\nabla^{2} \Phi+\frac{1}{p(X)} \frac{d p}{d X} \frac{\partial \Phi}{\partial X}+\frac{1}{q} \frac{d q}{d Y} \frac{\partial \Phi}{\partial X}\right]=0 .
\end{aligned}
$$

Multiplying equation (2.13) by $\frac{\lambda_{11}^{0}}{e_{15}^{0}}$ and equation (2.14) by $\frac{e_{15}^{0}}{c_{44}^{0}}$ and adding both we find that

$$
\left(\frac{\lambda_{11}^{0}}{e_{15}^{0}}+\frac{e_{15}^{0}}{c_{44}^{0}}\right)\left(\nabla^{2} u_{Z}+\frac{1}{p(X)} \frac{d p}{d X} \frac{\partial u_{Z}}{\partial X}+\frac{1}{q} \frac{d q}{d Y} \frac{\partial u_{Z}}{\partial Y}\right)=\frac{\rho_{0}}{e_{15}^{0}} \frac{\lambda_{11}^{0}}{c_{44}^{0}} \frac{\partial^{2} u_{Z}}{\partial t^{2}} .
$$

The above equation (2.15) can be written in the form

$$
\nabla^{2} u_{Z}+\frac{1}{p(X)} \frac{d p}{d X} \frac{\partial u_{Z}}{\partial X}+\frac{1}{q} \frac{d q}{d Y} \frac{\partial u_{Z}}{\partial Y}=\frac{1}{C^{2}} \frac{\partial^{2} u_{Z}}{\partial t^{2}}
$$

where

$$
C=\sqrt{\frac{\mu}{\rho_{0}}}, \quad \mu=c_{44}^{0}+\frac{\left(e_{15}^{0}\right)^{2}}{\lambda_{11}^{0}} .
$$

We now introduce a new function $\Psi$ by the relation

$$
\Phi=\Psi+\frac{e_{15}^{0}}{\lambda_{11}^{0}} u_{Z}
$$

and substitute for $\Phi$ into equation (2.14) to find that

$$
\nabla^{2} \Psi+\frac{1}{p(X)} \frac{d p}{d X} \frac{\partial \Psi}{\partial X}+\frac{1}{q} \frac{d q}{d Y} \frac{\partial \Psi}{\partial Y}=0 .
$$


For the problem of a moving surface with constant velocity $V$ along the $X$ direction, it is convenient to introduce a Galilean transformation such as

$$
x=X-V t, y=Y, z=Z,
$$

where $(x, y, z)$ is the translating coordinate system attached to the moving surface.

In the transformed coordinate system, by making use of (2.20), the equations (2.16) and (2.19) can be written in the form

$$
\begin{array}{r}
\nabla^{2} u_{z}+\frac{1}{p(x)} \frac{d p}{d x} \frac{\partial u_{z}}{\partial x}+\frac{1}{q(y)} \frac{d q}{d y} \frac{\partial u_{z}}{\partial y}-\frac{V^{2}}{C^{2}} \frac{\partial^{2} u_{z}}{\partial x^{2}}=0, \\
\nabla^{2} \Psi+\frac{1}{p(x)} \frac{d p}{d x} \frac{\partial \Psi}{\partial x}+\frac{1}{q(y)} \frac{d q}{d y} \frac{\partial \Psi}{\partial y}=0,
\end{array}
$$

where we assume that

$$
\frac{1}{p(X)} \frac{d p}{d X}=\frac{1}{p(x)} \frac{d p}{d x}
$$

Assuming

$$
\Psi=\frac{F(x, y)}{\sqrt{p(x) q(y)}},
$$

we find that equation (2.22) becomes

$$
\nabla^{2} F-\left[\left(\frac{1}{2 q} \frac{d^{2} q}{d y^{2}}-\frac{1}{4}\left(\frac{1}{q} \frac{d q}{d y}\right)^{2}\right)+\left(\frac{1}{2 p} \frac{d^{2} p}{d x^{2}}-\frac{1}{4}\left(\frac{1}{p} \frac{d p}{d x}\right)^{2}\right)\right] F=0 .
$$

If we assume

$$
\begin{aligned}
& F(x, y)=X(x) Y(y), \\
& \frac{1}{2 p} \frac{d^{2} p}{d x^{2}}-\frac{1}{4}\left(\frac{1}{p} \frac{d p}{d x}\right)^{2}=a_{0}, \\
& \frac{1}{2 q} \frac{d^{2} q}{d y^{2}}-\frac{1}{4}\left(\frac{1}{q} \frac{d q}{d y}\right)^{2}=b_{0},
\end{aligned}
$$

where $a_{0}$ and $b_{0}$ are constants, we find from (2.25) that

$$
\begin{aligned}
& \frac{d^{2} X}{d x^{2}}+\left[k^{2}-a_{0}\right] X=0, \\
& \frac{d^{2} Y}{d y^{2}}-\left[k^{2}+b_{0}\right] Y=0,
\end{aligned}
$$

where $-k^{2}$ is a separation constant. Making use of the solution to equations (2.29) and (2.30) we find from equation (2.26) and (2.24) that the solution of equation (2.22) is given by

$$
\Psi=\frac{1}{\sqrt{p(x) q(x)}} \int_{-\infty}^{\infty} e^{i \xi x} B(\xi) e^{-\left(\xi^{2}+a_{0}+b_{0}\right)^{\frac{1}{2}} y} d \xi
$$

where $B(\xi)$ is an unknown function. Assuming the solution of (2.21) in the form

$$
u_{z}(x, y, t)=X_{1}(x) Y_{1}(y)
$$


we find that

$$
\begin{aligned}
& \frac{d^{2} X_{1}}{d x^{2}}+\frac{\gamma}{p} \frac{d p}{d x} \frac{d X_{1}}{d x}-\gamma k_{1}^{2} X_{1}=0 \\
& \frac{d^{2} Y_{1}}{d y^{2}}+\frac{1}{q} \frac{d q}{d y} \frac{d Y_{1}}{d y}+k_{1}^{2} Y_{1}=0
\end{aligned}
$$

where

$$
\gamma=\frac{1}{\left(1-\frac{V^{2}}{C^{2}}\right)}
$$

and $k_{1}^{2}$ is a separation constant. The solution of equation (2.33) can be written in the form

$$
X_{1}=\frac{W}{[p(x)]^{\frac{\gamma}{2}}} .
$$

Substituting equation (2.36) into equation (2.33) we find that

$$
\frac{d^{2} W}{d x^{2}}+W\left[\left(\frac{\gamma}{2}-\frac{\gamma^{2}}{4}\right)\left(\frac{1}{p} \frac{d p}{d x}\right)^{2}-\frac{\gamma}{2 p} \frac{d^{2} p}{d x^{2}}-k_{1}^{2} \gamma\right]=0 .
$$

If we assume that

$$
\left(\frac{\gamma}{2}-\frac{\gamma^{2}}{4}\right)\left(\frac{1}{p} \frac{d p}{d x}\right)^{2}-\frac{\gamma}{2 p} \frac{d^{2} p}{d x^{2}}=c^{2}
$$

then equation (2.37) can be written in the form

$$
\frac{d^{2} W}{d x^{2}}+\left[c^{2}-k_{1}^{2} \gamma\right] W=0
$$

If we take

$$
W=e^{i x \xi}
$$

then from equation (2.39) we find that

$$
k_{1}^{2}=\left(\frac{c^{2}-\xi^{2}}{\gamma}\right) .
$$

Now if we assume that the solution of the differential equation (2.34) can be written in the form

then we find that

$$
Y_{1}=\frac{W_{1}}{\sqrt{q}}
$$

$$
\frac{d^{2} W_{1}}{d y^{2}}+\left[k_{1}^{2}-b_{0}\right] W_{1}=0 .
$$

From equations (2.41) and (2.43) we find that

$$
\frac{d^{2} W_{1}}{d y^{2}}-d_{0}^{2} W_{1}=0
$$

where

$$
d_{0}^{2}=b_{0}+\left(\frac{\xi^{2}-c^{2}}{\gamma}\right) .
$$

From equations (2.42) and (2.44) we obtain

$$
Y_{1}=\frac{A(\xi) e^{-d_{0} y}}{\sqrt{q}},
$$


and from equations (2.36) and (2.40) we find that

$$
X_{1}=\frac{e^{i x \xi}}{(p)^{\gamma / 2}} .
$$

Using equations (2.32), (2.46) and (2.47) we find that

$$
u_{z}(x, y, t)=X_{1} Y_{1}=\frac{1}{p^{\gamma / 2} q^{1 / 2}} \int_{-\infty}^{\infty} A(\xi) e^{i x \xi-d_{0} y} d \xi
$$

From equations (2.18), (2.31) and (2.48) we find the electric potential

$$
\begin{aligned}
& \Phi(x, y, t)=\frac{1}{\sqrt{p q}} \int_{-\infty}^{\infty} B(\xi) e^{i x \xi-\left(\xi^{2}+a_{0}+b_{0}\right)^{\frac{1}{2}} y} d \xi \\
& +\frac{e_{15}^{0}}{\lambda_{11}^{0} p^{\gamma / 2} q^{1 / 2}} \int_{-\infty}^{\infty} A(\xi) e^{i x \xi-d_{0} y} d \xi .
\end{aligned}
$$

In particular if we assume

$$
\left(c_{44}, e_{15}, \lambda_{11}, \rho\right)=\left(c_{44}^{0}, e_{15}^{0}, \lambda_{11}^{0}, \rho_{0}\right) e^{\alpha X+\beta Y},
$$

then from equations (2.20), (2.23), (2.27), (2.28), (2.38), and (2.45) we find that

$$
\left.\begin{array}{c}
p(X)=e^{\alpha X}, p(x)=e^{\alpha x+V \alpha t}, q(y)=e^{\beta y}, \\
a_{0}=\frac{\alpha^{2}}{4}, \quad b_{0}=\frac{\beta^{2}}{4}, \quad c^{2}=-\frac{\gamma^{2} \alpha^{2}}{4}, \\
k_{1}^{2}=-\frac{1}{\gamma}\left(\frac{\gamma^{2} \alpha^{2}}{4}+\xi^{2}\right), \quad d_{0}=\left(\frac{\beta^{2}}{4}+\frac{\xi^{2}-c^{2}}{\gamma}\right)^{\frac{1}{2}}
\end{array}\right\} .
$$

In this case from equations (2.48) and (2.49) we find that

$$
\begin{aligned}
& u_{z}(x, y, t)=\frac{1}{\sqrt{e^{\alpha x \gamma+V t \gamma \alpha+\beta y}}} \int_{-\infty}^{\infty} A(\xi) e^{i x \xi-d_{0} y} d \xi, \\
& \Phi(x, y, t)=\frac{1}{\sqrt{e^{\alpha x+\alpha V t+\beta y}}} \int_{-\infty}^{\infty} B(\xi) e^{i x \xi-\left(\xi^{2}+\frac{\alpha^{2}+\beta^{2}}{4}\right)^{\frac{1}{2}} y} d \xi \\
& +\frac{e_{15}^{0}}{\lambda_{11}^{0} \sqrt{e^{\alpha x \gamma+\alpha \gamma V t+\beta y}}} \int_{-\infty}^{\infty} A(\xi) e^{i x \xi-d_{0} y} d \xi,
\end{aligned}
$$

where $d_{0}$ is given by equation (2.52).

3. Application. We apply the above solution to the following moving contact problem.

We assume that a line load moves with a constant velocity $V$ over a piezoelectric half-space for an infinitely long time. The boundary conditions for the moving contact problem in moving coordinates are

$$
u_{z}(x, 0, t)=f(x, t), \Phi(x, 0, t)=g(x, t),-\infty<x<\infty, 0 \leq t,
$$

where $f(x, t)$ and $g(x, t)$ are prescribed functions satisfying $f(x, t) \neq 0$ and $g(x, t) \neq 0$.

In the literature on piezoelectric materials the boundary conditions (3.1) are well known for the contact problem. When we compare the boundary conditions (3.1) for moving contact with moving crack, then it can resemble neither that of a permeable nor an impermeable condition of crack surfaces. Crack contains two surfaces of piezoelectric material in which a permeable or an impermeable condition exists, while the geometry of 
contact is different than that of the crack case. In the contact problem there is no piezoelectric material above the contact region, while in the crack problem the piezoelectric material exists above as well as below the crack surface. In a moving contact problem the permeable conditions at $y=0$ and $y=+0$ can exist.

Making use of equations (2.53), (2.54) and (3.1) and the Fourier transforms we find that

$$
\begin{aligned}
& A(\xi)=\left(\frac{1}{2 \pi}\right) \int_{-\infty}^{\infty} f(x, t) e^{-i x \xi+\frac{\alpha \gamma}{2}(x+V t)} d x \\
& B(\xi)=\left(\frac{1}{2 \pi}\right) \int_{-\infty}^{\infty}\left[g(x, t)-\frac{e_{15}^{0}}{\lambda_{11}^{0}} f(x, t)\right] e^{-i x \xi+\frac{\alpha}{2}(x+V t)} d x .
\end{aligned}
$$

From equations (2.53), (2.54), (3.2) and (3.3) we can write

$$
\begin{aligned}
& u_{z}(x, y, t)=\frac{1}{2 \pi \sqrt{e^{\alpha \gamma x+\beta y}}} \int_{-\infty}^{\infty} e^{i x \xi-d_{0} y} d \xi \int_{-\infty}^{\infty} f(u, t) e^{\left(-i \xi+\frac{\alpha \gamma}{2}\right) u} d u \\
& \Phi(x, y, t)=\frac{e_{15}^{0}}{2 \pi \lambda_{11}^{0}} \frac{1}{\sqrt{e^{\alpha \gamma x+\beta y}}} \int_{-\infty}^{\infty} e^{i x \xi-d_{0} y} d \xi \int_{-\infty}^{\infty} f(u, t) e^{\left(-i \xi+\frac{\alpha \gamma}{2}\right) u} d u \\
& +\frac{1}{2 \pi} \frac{1}{\sqrt{e^{\alpha x+\beta y}}} \int_{-\infty}^{\infty} e^{i x \xi-\left(\xi^{2}+\frac{\alpha^{2}+\beta^{2}}{4}\right)^{\frac{1}{2}} y} d \xi \\
& \times \int_{-\infty}^{\infty}\left[g(u, t)-\frac{e_{15}^{0}}{\lambda_{11}^{0}} f(u, t)\right] e^{\left(-i \xi+\frac{\alpha}{2}\right) u} d u .
\end{aligned}
$$

With the help of equations (3.4) and (3.5) we find that

$$
\begin{aligned}
& \sigma_{y z}(x, y, t)=-\frac{1}{2 \pi}\left(c_{44}^{0}+\frac{\left(e_{15}^{0}\right)^{2}}{\lambda_{11}^{0}}\right) e^{\alpha x\left(1-\frac{\gamma}{2}\right)+\frac{\beta}{2} y+V \alpha t} \\
& \times \int_{-\infty}^{\infty}\left(d_{0}+\frac{\beta}{2}\right) e^{i x \xi-d_{0} y} d \xi \int_{-\infty}^{\infty} f(u, t) e^{\left(-i \xi+\frac{\alpha \gamma}{2}\right) u} d u \\
& -\frac{e_{15}^{0}}{4 \pi}\left[\beta+\left(4 \xi^{2}+\alpha^{2}+\beta^{2}\right)^{\frac{1}{2}}\right] e^{\frac{1}{2}(\alpha x+\beta y)+\alpha V t} \\
& \times \int_{-\infty}^{\infty} e^{i x \xi-\left(\xi^{2}+\frac{\alpha^{2}+\beta^{2}}{4}\right)^{\frac{1}{2}} y} d \xi \int_{-\infty}^{\infty}\left[g(u, t)-\frac{e_{15}^{0}}{\left.\lambda_{11}^{0} f(u, t)\right] e^{\left(-i \xi+\frac{\alpha}{2}\right) u} d u .}\right.
\end{aligned}
$$

For particular values of $f(x, t)$ and $g(x, t)$, the integrals in equations (3.4), (3.5) and (3.6) can be evaluated to obtain closed form expressions for $u_{z}(x, y, t), \Phi(x, y, t)$ and

$\sigma_{y z}(x, y, t)$.

\section{REFERENCES}

[1] Zhu, X., Wang, Q. and Meng, Z., A functionally graded piezoelectric actuator prepared by metallurgical process in PMN-PZ-PT system. J. Mater. Sci. Lett. 14 (1995), pp. 516-518.

[2] Zhu, Z., Zhu, J., Zhou, S., Li, Q. and Liu, Z., Microstructures of the monomorph piezoelectric ceramic actuators with functionally gradient sensor. Actuators A74 (1999), pp. 198-202.

[3] Wang B. L., A mode III crack in functionally graded piezoelectric materials. Mech. Res. Commun. 30 (2003), pp. 151-159.

[4] Wang B. L. and Zhang, X. H., A mode III crack in a functionally graded piezoelectric material strip. ASME J. Appl. Mech. 71 (2004) pp. 327-333.

[5] Li, Chunya and Weng, G. J., Antiplane Crack Problem in Functionally Graded Piezoelectric Materials. ASME, J. Appl. Mech. 69 (2002) pp. 481-488. 
[6] Wu, C. M., Kahn, M. and Moy W., Piezoelectric ceramics with functional gradients, a new application in material design. J. Am. Ceramic Soc. 79 (1996) pp. 809-812.

[7] Lee, P. C. Y. and Yu, J. D., Governing equations for a piezoelectric plate with graded properties across the thickness. IEEE Trans. Ultrason. Ferroelectr. Freq Control 45 (1998) pp. 236-250.

[8] Hu, K. Q., Zhong, Z. and Jin, B., Anti-plane shear crack in a functionally gradient piezoelectric material. Acta Mech. Solida Sin. 15 (2002), pp. 140-148.

[9] Hu, K. Q., Zhong, Z. and Jin, B., Electroelastic intensification near anti-plane crack in a functionally gradient piezoelectric strip. Acta Mech. Solida Sin. 16 (2003), pp. 197-204.

[10] Hu, K. Q., Zhong, Z. and Jin, B., Anti-plane shear crack in a functionally gradient piezoelectric layer bonded to dissimilar half-spaces. Int. J. Mech. Sci. 47 (2005), pp. 82-93.

[11] Jin, B. and Zhong, Z., A moving mode-III crack in functionally graded piezoelectric material: permeable problem. Mech. Research Commun.29, (2002), pp. 217-224.

[12] Li, C., and Weng, G.J., Yoffe-type moving crack in a functionally graded piezoelectric material. Proc. R. Soc. Lond. A. 458, (2002), pp. 381-399. MR1889934 (2002m:74046)

[13] Kwon, J. H., Lee, K. Y. and Kwon, S. M., Moving crack in a piezoelectric ceramic strip under anti-plane loading. Mech. Res. Commu. 27 (2000), pp. 327-332.

[14] Kwon, S. M. and Lee, K. Y., Constant moving crack in a piezoelectric block: anti-plane problem, Mech. Mater. 33 (2001), pp. 649-657.

[15] Kwon, S. M., Choi, H. S. and Lee, K. Y., Moving eccentric crack in a piezoelectric strip bonded to two elastic materials, Arch. Appl. Mech. 72 (2002) pp. 160-170.

[16] Kwon, S. M., Lee, J. S. and Lee, K. Y., Moving eccentric crack in a piezoelectric strip bonded to two elastic half-planes. Int. J. Solids Struct. 39 (2002), pp. 4395-4406.

[17] Lee, J. S., Kwon, S. M., Lee, K. Y., and Kwon, J. H., Anti-plane interfacial Yoffe-crack between piezoelectric and two orthotropic layers. Eur. J. Mech. A/Solids 21 (2002), pp. 483-492.

[18] Ikeda, T., Fundamentals of Piezoelectricity. Oxford University Press, Oxford, UK. (1996).

[19] Jaffe, B., Cook, Jr. W. R. and Jaffe, H., Piezoelectric Ceramics. Academic Press, London (1996).

[20] Borrelli, A., Hogan, C. O. and Patria, M. C., Exponential decay of end effects in anti-plane shear for functionally graded piezoelectric materials. Proc. Royal. Soc. London. A 460 (2004), pp. 1193-1212. MR2133863 (2005k:74032)

[21] Deng, Wei and Meguid, S. A., Analysis of conducting rigid inclusion at the interface between two dissimilar piezoelectric materials. ASME Journal of Applied Mechanics 65 (1998), pp. 76-83.

[22] Deeg, W. F., The analysis of dislocation, crack and inclusion problems in piezoelectric solids. $\mathrm{Ph}$. D. Thesis Stanford University, CA, (1980).

[23] Asundi, A. and Deng, W., Rigid inclusion on the interface between two bonded anisotropic media. J. Mech. Phys. Solids, 43 (1995), pp. 1045-1058

[24] Chen, T., The rotation of a rigid ellipsoidal inclusion embedded in an anisotropic piezoelectric medium. Int. J. Solids Structures 30 (1993), pp. 1983-1995. 